\title{
ON PROJECTIVE HJELMSLEV PLANES OF LEVEL $\boldsymbol{n}$
}

\author{
by G. HANSSENS $\dagger$ and H. VAN MALDEGHEM $\dagger$
}

(Received 28 March, 1988)

In this paper, we establish a new (but equivalent) definition of projective Hjelmslev planes of level $n$. This shows that the $n$th floor of a triangle building is a projective Hjelmslev plane of level $n$ (a result already announced in [9], but left unproved). This will allow us to characterize Artmann-sequences by means of their inverse limits and to construct new ones. We also deduce a new existence theorem for level $\boldsymbol{n}$ projective Hjelmslev planes. All results hold in the finite as well as in the infinite case.

\section{Preliminaries.}

Definition 1. An incidence structure $H=(P(H), L(H), I)$ is called a projective Hjelmslev plane (or briefly a PH-plane) if it satisfies (H.1), (H.2) and (H.3):

(H.1) there is at least one line joining any two points;

(H.2) there is at least one point on any two lines;

(H.3) there is a canonical epimorphism $\alpha_{H}: H \rightarrow \mathscr{P}_{H}$ with $\mathscr{P}_{H}$ a non-degenerate projective plane, such that $\alpha_{H}(X)=\alpha_{H}(Y)$ if and only if either $X, Y \in L(H)$ and $X$ and $Y$ join more than one point, or $X, Y \in P(H)$ and $X$ and $Y$ are on more than one common line, for all $X, Y \in P(H) \cup L(H)$.

Definition 2 (Definition by induction on $n$ ). A PH-plane of level $n$ is a structure $\mathscr{H}_{n}=\left(H_{n}, H_{n-1}, \ldots, H_{1}, \alpha_{n-1}^{n}, \ldots, \alpha_{1}^{2}\right)$ such that

(i) $H_{1}$ is a non-degenerate projective plane and $H_{n}$ is a PH-plane;

(ii) $\left(H_{n-1}, \ldots, H_{1}, \alpha_{n-2}^{n-1}, \ldots, \alpha_{1}^{2}\right)$ is a PH-plane of level $n-1$;

(iii) $\alpha_{n-1}^{n}: H_{n} \rightarrow H_{n-1}$ is an epimorphism of PH-planes;

(iv) the following conditions (V), (Ma), (Mb), (Mc) and (N) are satisfied.

(V) $\mathscr{P}_{H_{n}}=\mathscr{P}_{H_{n-1}}$ and $\alpha_{H_{n-1}} \circ \alpha_{n-1}^{n}=\alpha_{H_{n}}$.

(Ma) If $P, Q \in P\left(H_{n}\right), \quad L, M \in L\left(H_{n}\right), \quad Q I L I P I M, \quad \alpha_{n-1}^{n}(P)=\alpha_{n-1}^{n}(Q)$ and $\alpha_{H_{n}}(L)=\alpha_{H_{n}}(M)$, then $Q I M$.

(Mb) The dual statement of (Ma).

(Mc) There exist distinct points $P, Q \in P\left(H_{n}\right)$ such that $\alpha_{n-1}^{n}(P)=\alpha_{n-1}^{n}(Q)$ and dually.

The epimorphism $\alpha_{j}^{n}: H_{n} \rightarrow H_{j}$ is defined by $\alpha_{j}^{n}=\alpha_{j}^{j+1} \circ \alpha_{j+1}^{j+2} \circ \ldots \circ \alpha_{n-1}^{n}$ for $1 \leq j<n$, and $\alpha_{n}^{n}$ is the identity on $H_{n}$. Note that $\alpha_{H_{n}}=\alpha_{1}^{n}$.

We define an equivalence relation $(\sim j)$ by $P(\sim j) Q$ if $\alpha_{j}^{n}(P)=\alpha_{j}^{n}(Q)$, for all $P, Q \in P\left(H_{n}\right), j<n$ and by definition $P(\sim 0) Q$ always. Similarly for lines.

(N) For all $L, M \in L\left(H_{n}\right)$, we have $L(\sim j) M$ if and only if $Q I M$ for all $Q \in P\left(H_{n}\right)$ such that $Q I L$ and $P(\sim n-j) Q$ for some $P \in P\left(H_{n}\right)$ with $L I P I M$.

$\dagger$ This research was supported by the National Fund for Scientific Research (N.F.W.O.) of Belgium.

Glasgow Math. J. 31 (1989) 257-261. 
Definitions 1 and 2 are taken from Artmann [1] and [2].

Definition 3. An Artmann-sequence $\left(H_{n}, \alpha_{n}^{n+1}\right)_{n \in \mathbb{N}^{*}}$ is an infinite sequence of PH-planes together with epimorphisms $\alpha_{n}^{n+1}: H_{n+1} \rightarrow H_{n}$ such that $\left(H_{n}, H_{n-1}, \ldots\right.$, $\left.H_{1}, \alpha_{n-1}^{n}, \ldots, \alpha_{1}^{2}\right)$ is a PH-plane of level $n$ for each $n$.

B. Artmann showed in [2] that there exists an Artmann-sequence $\left(H_{n}, \alpha_{n}^{n+1}\right)_{n \in \mathbb{N}^{*}}$ for every projective plane $H_{1}$.

Besides the notions of projective plane, affine plane and dual affine plane, the following notion will be useful (see [8]).

DefinItion 4. Suppose $\mathscr{P}$ is a projective plane and $(P, L)$ is an incident point-line pair of $\mathscr{P}$. The incidence structure $\mathscr{H}$ obtained from $\mathscr{P}$ by deleting all lines incident with $P$ and all points incident with $L$ is called a helicopter plane.

Suppose $\mathscr{H}_{n}=\left(H_{n}, \ldots, H_{1}, \alpha_{n-1}^{n}, \ldots, \alpha_{1}^{2}\right)$ is a PH-plane of level $n$. We remark that (N) implies that every line of $H_{n}$ is completely determined by the set of points incident with it. Hence we can identify every line with that set. Now let $P \in P\left(H_{n}\right)$; we denote by $\bar{P}^{i}, 0 \leq i \leq n$, the set $\left\{Q \in P\left(H_{n}\right) \mid P(\sim n-i) Q\right\}$. We define $\bar{B}_{n}^{i}=\left\{L \cap \bar{P}^{i} \mid L \in\right.$ $\left.L\left(H_{n}\right), P \in P\left(H_{n}\right), P I L\right\}$ for $0 \leq i \leq n$. Now fix $i, 0 \leq i \leq n-1$, and $b \in \bar{B}_{n-1}^{i}$. We define an incidence structure $S_{b}=\left(P\left(S_{b}\right), L\left(S_{b}\right), I\right)$ as follows.

$$
\begin{aligned}
& L\left(S_{b}\right)=\left\{c \in \bar{B}_{n}^{i+1} \mid \alpha_{n-1}^{n}(c)=b\right\}, \\
& P\left(S_{b}\right)=\left\{c \cap \bar{P}^{i} \mid c \in L\left(S_{b}\right), P \in c\right\}, \\
& c I c^{\prime} \text { if and only if } c^{\prime} \subseteq c, \text { for all } c \in L\left(S_{b}\right) \text { and } c^{\prime} \in P\left(S_{b}\right) .
\end{aligned}
$$

From Artmann [1, Satz 1], it follows that $S_{b}$ is an affine plane if $b \in \bar{B}_{n-1}^{0}$ and a dual affine plane if $b \in \bar{B}_{n-1}^{n-1}$.

\section{Main Results.}

TheOREM. A series of PH-planes $H_{n}, H_{n-1}, \ldots, H_{1}$ together with epimorphisms $\alpha_{j}^{j+1}: H_{j+1} \rightarrow H_{j}$ for $j=1, \ldots, n-1$ form a PH-plane of level $n,\left(H_{n}, H_{n-1}, \ldots, H_{1}\right.$, $\left.\alpha_{n-1}^{n}, \ldots, \alpha_{1}^{2}\right)$, if and only if they satisfy $(\mathrm{G} .1)_{n},(\mathrm{G} .2)_{n}$ and (G.3) $)_{n}$ below.

(G.1 $)_{n}\left|\left(\alpha_{j}^{j+1}\right)^{-1}(X)\right|>1$ for all points and lines $X$ in $H_{j}$ and all $j$ with $1 \leq j<n$.

Suppose $X, Y \in P\left(H_{n}\right)$ or $X, Y \in L\left(H_{n}\right)$ and let $\alpha_{j}^{n}=\alpha_{j}^{j+1} \circ \alpha_{j+1}^{j+2} \circ \ldots \circ \alpha_{n-1}^{n}, j<n$ and $\alpha_{n}^{n}$ be the identity map in $H_{n}$. We write $u(X, Y)=j$ if $\alpha_{j}^{n}(X)=\alpha_{j}^{n}(Y)$ and $\alpha_{j+1}^{n}(X) \neq$ $\alpha_{j+1}^{n}(Y)$. Also, $u(X, Y)=n$ if $X=Y$. If $P \in P\left(H_{n}\right)$ and $L \in L\left(H_{n}\right)$, then we write $u(P, L)=j$ if $\alpha_{j}^{n}(P) I \alpha_{j}^{n}(L)$ and $\alpha_{j+1}^{n}(P) \mathrm{I} \alpha_{j+1}^{n}(L) ; u(P, L)=n$ if $P I L$.

(G.2) $)_{n}$ If $P, Q \in P\left(H_{n}\right), L, M \in L\left(H_{n}\right)$ and $0 \leq k \leq \inf \{u(Q, L), u(P, L), u(P, M)\}$, then

(i) there is at least one line joining $P$ and $Q$ and there is at least one point on both $L$ and $M$,

(ii) $u(Q, M) \geq k$ if and only if $u(Q, P)+u(L, M) \geq k$.

$(\mathrm{G} .3)_{n} H_{1}$ is a non-degenerate projective plane. 
Corollary 1. Suppose $\mathscr{H}_{n}$ is a PH-plane of level $n$. If $b \in \bar{B}_{n-1}^{i}, 0<i<n-1$, then $S_{b}$ as defined at the end of Section 1 is a helicopter plane.

Corollary 2. Suppose $\left(H_{n}, \alpha_{n}^{n+1}\right)_{n \in \mathbb{N}^{*}}$ is an Artmann-sequence with inverse limit $H_{\infty}$. Then $H_{\infty}$ is a projective plane. Let $(R, T)$ be any coordinatizing PTR of $H_{\infty}$ (see [5] for the definition); then there exists a surjective map $v: R^{2} \rightarrow Z \cup\{+\infty\}$ satisfying

(v.1) $v(a, b)=+\infty$ if and only if $a=b$, for all $a, b \in R$,

(v.2) $v(a, c) \geq \inf \{v(a, b), v(b, c)\}$ and if $v(a, b) \neq v(b, c)$, equality holds, for all $a, b, c \in R$,

(v.3) if $T\left(a_{1}, b_{1}, c_{1}\right)=T\left(a_{1}, b_{2}, c_{2}\right)$ and $T\left(a_{2}, b_{1}, c_{1}\right)=T\left(a_{2}, b_{2}, c_{3}\right)$, then $v\left(a_{1}, a_{2}\right)+$ $v\left(b_{1}, b_{2}\right)=v\left(c_{2}, c_{3}\right)$.

Conversely, if $\mathscr{P}$ is a projective plane coordinatized by a $\operatorname{PTR}(R, T)$ admitting a surjective map $v$ as above, then $\mathscr{P}$ is isomorphic to the inverse limit of some Artmann-sequence.

COROLlaRY 3. Let $q$ be the order of a projective plane, possibly infinite. Let $\Gamma$ be the set of all projective planes of order $q$. Then an Artmann-sequence $\left(H_{n}, \alpha_{n}^{n+1}\right)_{n \in \mathbb{N}^{*}}$ can be constructed step by step which satisfies the following conditions.

(i) $H_{1}$ is any element of $\Gamma$, chosen in advance.

(ii) If the level $n$ PH-plane $\left(H_{n}, \ldots, H_{1}, \alpha_{n-1}^{n}, \ldots, \alpha_{1}^{2}\right)$ has already been constructed, then $H_{n+1}$ and the epimorphism $\alpha_{n}^{n+1}$ can be constructed in such $a$ way that $\left(H_{n+1}, H_{n}, \ldots, H_{1}, \alpha_{n}^{n+1}, \alpha_{n-1}^{n}, \ldots, \alpha_{1}^{2}\right)$ becomes a PH-plane of level $n+1$ and the following conditions are satisfied. For each $i=0,1, \ldots, n$, and each $b \in \bar{B}_{n}^{i}$, let $\mathscr{P}_{b}$ be any prescribed element of $\Gamma$. Then $S_{b}$ is any prescribed helicopter plane, affine plane or dual affine plane arising from $\mathscr{P}_{b}$ according to whether $0<i<n, i=n$ or $i=0$.

\section{Proofs.}

Proof of the theorem. We proceed by induction on $n \in \mathbb{N}^{*}$. The statement is trivial for $n=1$. So suppose $n>1$. We remark that (G.1) $)_{n},(\mathrm{G} .2)_{n}$ and (G.3) imply (G.1) $)_{n-1}$, (G.2) $)_{n-1}$ and (G.3) $)_{n-1}$ for $H_{n-1}, \ldots, H_{1}$ with the epimorphisms $\alpha_{j}^{j+1}$.

(I) Assume $H_{n}, \ldots, H_{1}, \alpha_{j}^{j+1}(1 \leq j \leq n-1)$ are given satisfying (G.1) ${ }_{n},(\mathrm{G} .2)_{n}$ and (G.3) ${ }_{n}$. The conditions (H.1) and (H.2) follow directly from (G.2) ${ }_{n}$ (i). We now show (H.3). Suppose $L, M \in L\left(H_{n}\right)$ and let $\mathscr{P}_{H_{n}}=H_{1}$ and $\alpha_{H_{n}}=\alpha_{1}^{n}$. Suppose first $\alpha_{H_{n}}(L)=$ $\alpha_{H_{n}}(M)$, so $u(L, M) \geq 1$. Let $P \in P\left(H_{n}\right)$ be incident with both $L$ and $M$. Let $Q \in P\left(H_{n}\right)$ be such that $u(P, Q)=n-1$ (hence $P \neq Q$ ) and $Q I P(Q$ exists by $[8, \S 6.1 .1]$ ). Applying (G.2) $)_{n}$ (ii) for $k=n$, we obtain $u(Q, M) \geq n$, hence $Q I M$. Suppose now $\alpha_{H_{n}}(L) \neq \alpha_{H_{n}}(M)$, so $u(L, M)=0$. If $P, Q \in P\left(H_{n}\right)$ are incident with both $L$ and $M$, then applying (G.2) ${ }_{n}$ (ii) for $k=n$, we obtain $u(P, Q) \geq n$, hence $P=Q$. Similarly, one shows the dual. This proves (H.3).

When one remarks that $P(\sim j) Q$ if and only if $u(P, Q) \geq j$ for $P, Q \in P\left(H_{n}\right)$ and similarly for lines, the axioms (V), (Ma), (Mb) and (Mc) become trivial to verify. We now check (N). The "if"-part follows from (G.2) ${ }_{n}$ (ii) for $k=n$. We now show the "only if'-part. Suppose $L, M \in L\left(H_{n}\right), P \in P\left(H_{n}\right)$ with $L I P I M$. Let $P^{*} \in P\left(H_{n}\right)$ be such that $u\left(P, P^{*}\right)=n-j\left(P^{*}\right.$ exists by $\left.(\mathrm{G} .1)_{n}\right)$. Suppose first $u\left(P^{*}, L\right)>n-j$. Let $Q^{*}$ be a point 
such that $u\left(Q^{*}, L\right)=0\left(Q^{*}\right.$ is any element in the inverse image under $\alpha_{H_{n}}$ of any point of $H_{1}$ not incident with $\left.\alpha_{H_{n}}(L)\right)$. Consider any line $L^{*} \in L\left(H_{n}\right)$ incident with both $P^{*}$ and $Q^{*}$. Since $Q^{*} I L^{*}, u\left(L, L^{*}\right)=0$. Consider the unique point $Q \in P\left(H_{n}\right)$ incident with both $L$ and $L^{*}$. Applying (G.2) (ii) on $P^{*} I L^{*} I Q I L$, we obtain $u\left(P^{*}, Q\right)=u\left(P^{*}, L\right)>n-j$. Hence $u(P, Q)=n-j$ and so $Q I M$. By $(\mathrm{G} .2)_{n}$ (ii) again, $L(\sim j) M$. Suppose now $u\left(P^{*}, L\right)=n-j$ (it cannot be smaller!). Consider any line $M^{*}$ incident with both $P$ and $P^{*}$. By $(\mathrm{G} .2)_{n}(\mathrm{ii}), u\left(L, M^{*}\right)=0$. Let $Q^{*}$ by any point such that $u\left(Q^{*}, L\right)=u\left(Q^{*}, M^{*}\right)=$ 0 (similar construction to the one above). Choose any line $L^{*}$ incident with both $P^{*}$ and $Q^{*}$. Let $Q \in P\left(H_{n}\right)$ be incident with both $L$ and $L^{*}$. In the same way as before, we obtain $n-j=u\left(P^{*}, L\right)=u\left(P^{*}, Q\right)=u\left(Q, M^{*}\right)=u(Q, P)$ and $u(L, M) \geq j$, hence $L(\sim j) M$ again.

(II) Assume, conversely, $\left(H_{n}, \ldots, H_{1}, \alpha_{n-1}^{n}, \ldots, \alpha_{1}^{2}\right)$ is a level $n$ PH-plane. We show (G.1) ${ }_{n}$. The existence of the sequence follows from (V) and (Mc). By Artmann [1, Satz 1.a], $\left|\left(\alpha_{j}^{j+1}\right)^{-1}(X)\right|>1$ if $j=n-1$, and by the induction hypothesis, this is also true for $j<n-1$. This shows (G.1) $)_{n}$. The condition (G.2) $n_{n}(\mathrm{i})$ is equivalent to (H.1) and (H.2). And (G.2) $\left(\right.$ ii) is an immediate consequence of (N) if $k=n$, and projecting onto $h_{k}$, $k<n$, (G.2) ${ }_{n}$ (ii) follows for all $k<n$. Finally, (G.3) ${ }_{n}$ follows from (H.3). This completes the proof of the theorem.

By [8], this theorem forges a quite unexpected link between two different worlds: the world of affine buildings and the world of PH-planes. It can give a new impulse to the study of the latter. Corollaries 1, 2 and 3 are three first examples of how properties of affine buildings may be translated to properties of level $n$ PH-planes.

Proof of Corollary 1. The axioms (G.1) $n_{n},(\mathrm{G} .2)_{n}$ and (G.3) ${ }_{n}$ are respectively equivalent to (PS), (RP) and (ND) of [8] and [9]. The result follows from [8, Proposition 6.1.10].

Proof of Corollary 2. The inverse limit $H_{\infty}$ is a projective plane by Artmann [2, Satz über den projektiven Limes]. By [9, Theorem (4.4.1)], $H_{\infty}$ is isomorphic to the geometry at infinity of some triangle building endowed with a maximal set of apartments (see Tits [7] for definitions). The result follows from [9, Theorem I]. The converse is a direct consequence of [9, Main Theorem and \$4.4] and the construction of triangle buildings in $[8]$.

Proof of Corollary 3. This is a consequence of Ronan's beautiful construction of buildings in [6].

Remark. Corollary 3 shows that the structure of level $n$ PH-planes is very "disconnected", in contrast to the impression one might have by considering the constructions of Artmann [2], Drake [4] and Cronheim [3]. In these constructions, wide classes of subgeometries of $H_{n}$ had to be chosen isomorphic. Note that Corollary 3 generalizes the constructions of Artmann [2] and Cronheim [3], but not Drake [4].

ACKNOWLEDGEMENT. We are very grateful to the referee for some very helpful remarks and suggestions regarding Sections 1 and 2 . 


\section{REFERENCES}

1. B. Artmann, Hjelmslev-Ebenen mit verfeinerten Nachbarschaftrelationen, Math. Z. 112 (1969), 163-180.

2. B. Artmann, Existenz und projektive Limiten von Hjelmslev-Ebenen $n$-ter Stufe, in Atti del Convegno di Geometria Combinatoria e sue Applicazioni, Perugia (1971), 27-41.

3. A Cronheim, Cartesian groups, formal power series and Hjelmslev-planes, Arch. Math. (Basel) 27 (1976), 209-220.

4. D. A. Drake, Construction of Hjelmslev planes, J. Geom. 10 (1977), 179-193.

5. D. R. Hughes and F. C. Piper, Projective planes (Springer-Verlag, 1972).

6. M. A. Ronan, A universal construction of buildings with no rank 3 residue of spherical type, in L. A. Rosati, ed., Buildings and the geometry of diagrams Proceedings Como 1984, Lecture Notes in Mathematics 1181, (Springer-Verlag, 1986), 242-248.

7. J: Tits, Immeubles de type affine, in L. A. Rosati, ed. Buildings and the geometry of diagrams Proceedings Como 1984, Lecture Notes in Mathematics 1181 (Springer-Verlag, 1986), 157-190.

8. H. Van Maldeghem, Non-classical triangle buildings, Geom. Dedicata 24 (1987), 123-206.

9. H. Van Maldeghem, Valuations on PTRs induced by triangle buildings, Geom. Dedicata 26 (1988), 29-84.

Seminarie voOr MeEtKunde en Kombinatoriek

RIJKSUNIVERSTTEIT VAN GENT

KRIJGSLAAN 281

B-9000 GENT

BELGIUM 